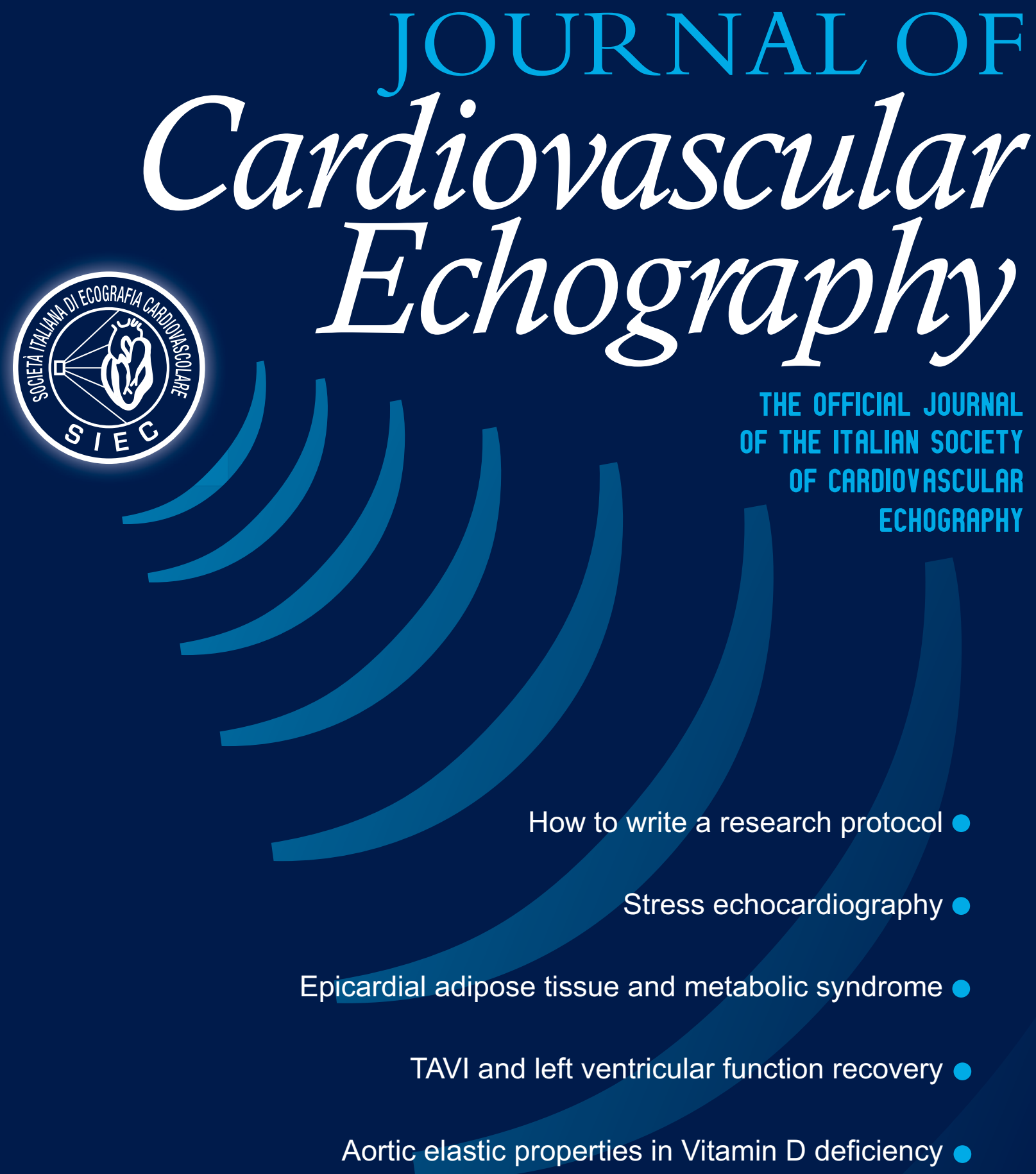

Reference values of systolic left and right function in neonates o 


\title{
Multimodality Imaging of Left Ventricular Clefts in an Asymptomatic Teenager
}

\author{
Chiara Bencini, Giovanni Corrado', Claudia Borghi², Stefano Carugo \\ Department of Cardiology, San Paolo Hospital, Milano State University, Milano, Departments of ${ }^{1}$ Cardiology and ${ }^{2}$ Radiology, Valduce Hospital, Como, Italy
}

\section{Abstract}

Left ventricular clefts (LVCs) are defined as deep, tight blood-filled invaginations within the ventricular myocardium localized predominantly in the basal posterior septum and LV-free wall. Usually, they are asymptomatic and incidentally discovered during diagnostic imaging procedures. LVC has been reported both in healthy volunteers and in patients affected with hypertrophic cardiomyopathy. Clinicians should be able to recognize LVC and to distinguish this entity from other myocardial wall defects with different pathological profile and clinical significance. We describe a case of multimodality imaging of multiple septal myocardial clefts in an asymptomatic teenager.

Keywords: Cardiac magnetic resonance, echocardiography, myocardial cleft

\section{INTRODUCTION}

Left ventricular clefts (LVCs) are congenital fissure-like protrusions penetrating more than $50 \%$ of the thickness of adjoining compacted myocardium which does not exceed beyond the myocardial margin. ${ }^{[1,2]}$ They have been reported in $5 \%-6 \%$ of the general population ${ }^{[1]}$ and are well detected by cardiac computerized tomography (CT) or magnetic resonance (MR); echocardiography is less sensitive and can fail their identification. ${ }^{[3]}$ LVC are most commonly located at the basal to mid inferoseptal and anteroseptal segment of the left ventricle, less commonly at mid inferior and apical septal segments. ${ }^{[3]}$ They contract normally without local hypokinesia or dyskinesia, usually obliterate during systole and are oriented approximately perpendicular to the long axis of the left ventricle. ${ }^{[1]}$ Some authors call this entity with different names such as recesses or crypts. The etiology of these myocardial structural defects is unclear. Some authors suggested that clefts represent early pathological alterations of the myocardium in patients with hypertrophic cardiomyopathy (HCM) mutations who had not yet developed echocardiographic evidence of hypertrophy, so they could represent a distinctive pathological expression of HCM. ${ }^{[3]}$ However, in large retrospective series in a general population studied with cardiac CT, LVC was found in $6.7 \%$

\begin{tabular}{|l|l|}
\hline \multicolumn{2}{|c|}{ Video available on: www.jcecho.org } \\
\hline \multicolumn{3}{|c|}{ Access this article online } \\
\hline Quick Response Code: & Website: \\
& www.jcecho.org \\
\cline { 2 - 2 } & DOI: \\
& 10.4103/jcecho.jcecho_10_18 \\
\hline
\end{tabular}

of patients who exhibited a good prognosis. As a matter of fact, on follow-up, there was no significant difference compared to the control group without LVC in the incidence of major adverse cardiac events or stroke. ${ }^{[3]}$ LVC must be distinguished from ventricular congenital aneurysm and ventricular diverticula, which have different histological and morphological characteristics and outcomes. Congenital left ventricular diverticulum is a malformation characterized by saccular protrusion extending beyond the borders of the myocardial margin, has a narrow communication with LV, and displays normal contraction. ${ }^{[3,4]}$ Congenital left ventricular aneurysms have a wide connection with the LV, are fibrotic, and usually akinetic or dyskinetic ${ }^{[5]}$ Congenital left ventricular diverticula and aneurysms have potentially harmful and even lethal complications; furthermore, they are frequently associated with structural cardiac anomalies. ${ }^{[4,5]}$

\section{Case Report}

An asymptomatic 17-year-old woman underwent echocardiogram at our laboratory because of heart murmur.

Address for correspondence: Dr. Chiara Bencini, Department of Cardiology, San Paolo Hospital, Asst Santi Paolo E Carlo, Via A Di Rudinì 8, 20142 Milano, Italy. E-mail: chiara.bencini@yahoo.it

This is an open access journal, and articles are distributed under the terms of the Creative Commons Attribution-NonCommercial-ShareAlike 4.0 License, which allows others to remix, tweak, and build upon the work non-commercially, as long as appropriate credit is given and the new creations are licensed under the identical terms.

For reprints contact: reprints@medknow.com

How to cite this article: Bencini $\mathrm{C}$, Corrado $\mathrm{G}$, Borghi $\mathrm{C}$, Carugo S. Multimodality imaging of left ventricular clefts in an asymptomatic teenager. J Cardiovasc Echography 2018;28:182-4. 

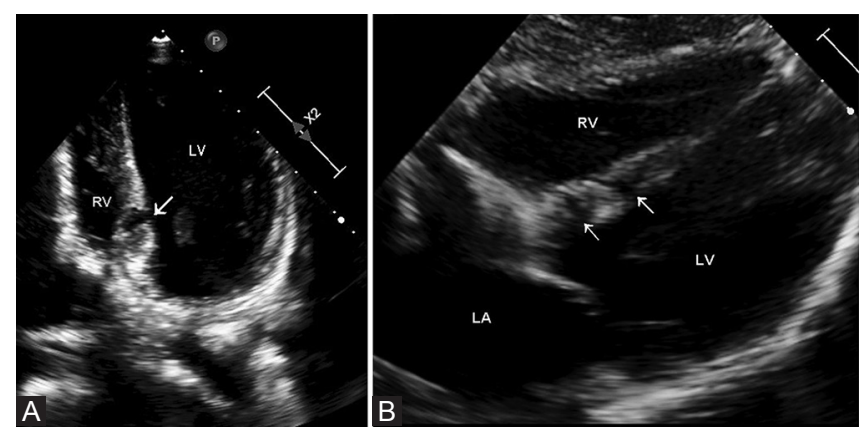

Figure 1: Two-dimensional transthoracic echo diastolic frames. Panel A: modified apical $4 \mathrm{c}$ chamber view. Panel B: subcostal view. White arrows indicate the entrance of the left ventricular clefts. In the subcostal view, both the basal and the more apical clefts are clearly visible. The more apical cleft bifurcates in its final part toward the right side of the interventricular septum. In 4-chamber view, only the most apical cleft is visible. During systole, do to myocardial contraction, blood is squeezed in the left ventricle and clefts are emptied and no more appreciable. $\mathrm{LA}=\mathrm{Left}$ atrium, $\mathrm{LV}=\mathrm{Left}$ ventricle, $\mathrm{RV}=$ Right ventricle

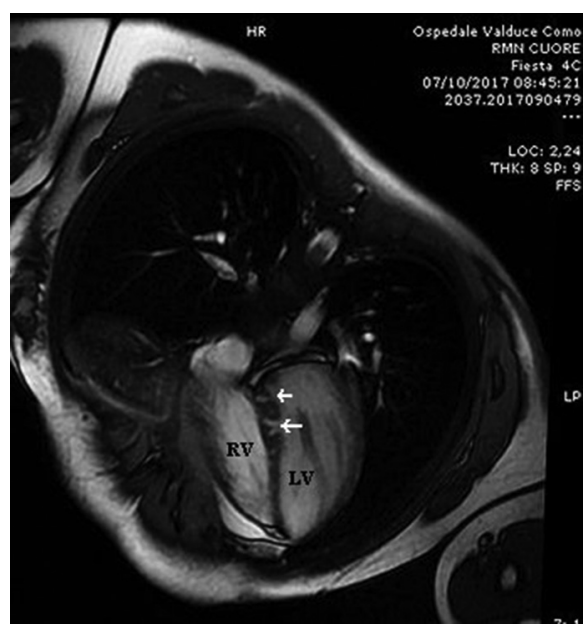

Figure 2: Cardiac magnetic resonance. Fast imaging employing steady state acquisition Two-dimensional - left ventricular 4-chamber view. Two myocardial clefts (white arrows) are visible in the basal inferior interventricular septum, contained by a normally contracting myocardium. As already visible in the echo imaging, the more apical cleft (longer arrow) bifurcates in its distal portion. Abbreviations as in Figure 1
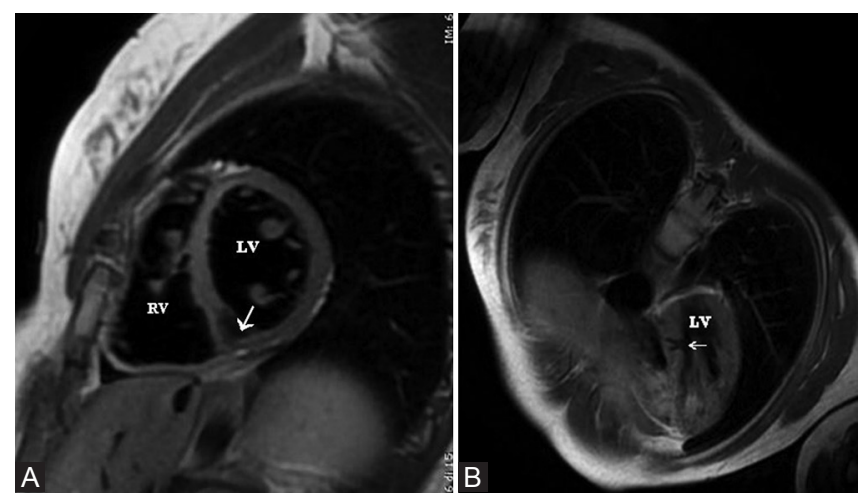

Figure 3: Cardiac magnetic resonance. Double inversion recovery. Left ventricular 4-chamber (Panel A) and short-axis view (Panel B). The bifurcated myocardial cleft in the inferior interventricular septum is depicted, contained by a normal myocardium (arrow). Abbreviations as in Figure 1
Rest electrocardiogram was normal. On family history, her father was affected by HCM. On echocardiography, we found normal volume, mass, and systodiastolic function of the LV. The right ventricle was normal too and no valve disease was detected. Nevertheless, we found two deep recesses in the basal and medium third of the interventricular septum containing luminal blood in diastole with complete obliteration in systole [Figure 1]. No interventricular shunt was found. The septal myocardium appeared to be compact, and no local hypertrophy was found. A cardiac MR confirmed the presence of two clefts of the septal myocardium penetrating more than $50 \%$ of the thickness of the septum, the distal one being bilobated [Figures 2 and 3]. The surrounding myocardium showed a normal contractility, as normal was the global systolic function of the left ventricle [Video 1]. Neither areas of fibrosis after gadolinium infusion nor markers of HCM were detected. The patient and the parents were reassured about the benign nature of these findings. Nevertheless, since some authors suggested that LVC may be more prevalent in HCM mutation carriers without hypertrophy ${ }^{[3]}$ and given that the father of the patient was affected with a clinically evident form of HCM, a genetic counseling was suggested.

LVC have been reported both in the general healthy population $^{[1]}$ and in HCM mutation carriers without LV hypertrophy. ${ }^{[3]}$ They must be correctly identified and diagnosed and should not be misinterpreted as ventricular diverticula or congenital aneurysms. Up to now, there are no robust data on the clinical significance of myocardial clefts in otherwise normal individuals. Nevertheless, on the basis of the existing literature, such individuals should be reassured and LVC should probably be regarded as incidental variants of myocardial structure unlikely to require further investigation and with an overall good prognosis on follow-up.

\section{Declaration of patient consent}

The authors certify that they have obtained all appropriate patient consent forms. In the form the patient(s) has/have given his/her/their consent for his/her/their images and other clinical information to be reported in the journal. The patients understand that their names and initials will not be published and due efforts will be made to conceal their identity, but anonymity cannot be guaranteed.

\section{Financial support and sponsorship}

Nil.

\section{Conflicts of interest}

There are no conflicts of interest.

\section{RefEREnCES}

1. Johansson B, Maceira AM, Babu-Narayan SV, Moon JC, Pennell DJ, Kilner PJ, et al. Clefts can be seen in the basal inferior wall of the left ventricle and the interventricular septum in healthy volunteers as well as patients by cardiovascular magnetic resonance. J Am Coll Cardiol 2007;50:1294-5.

2. Petryka J, Baksi AJ, Prasad SK, Pennell DJ, Kilner PJ. Prevalence of inferobasal myocardial crypts among patients referred for cardiovascular magnetic resonance. Circ Cardiovasc Imaging 2014;7:259-64. 
3. Germans T, Wilde AA, Dijkmans PA, Chai W, Kamp O, Pinto YM, et al. Structural abnormalities of the inferoseptal left ventricular wall detected by cardiac magnetic resonance imaging in carriers of hypertrophic cardiomyopathy mutations. J Am Coll Cardiol $2006 ; 48: 2518-23$
4. Tullu MS, Vaideeswar P, Deshmukh CT. Congenital left ventricular diverticula. Int J Cardiol 2000;73:293-5.

5. Du Toit HJ, Von Oppell UO, Hewitson J, Lawrenson J, Davies J. Left ventricular sub-valvar mitral aneurysms. Interact Cardiovasc Thorac Surg 2003;2:547-51. 